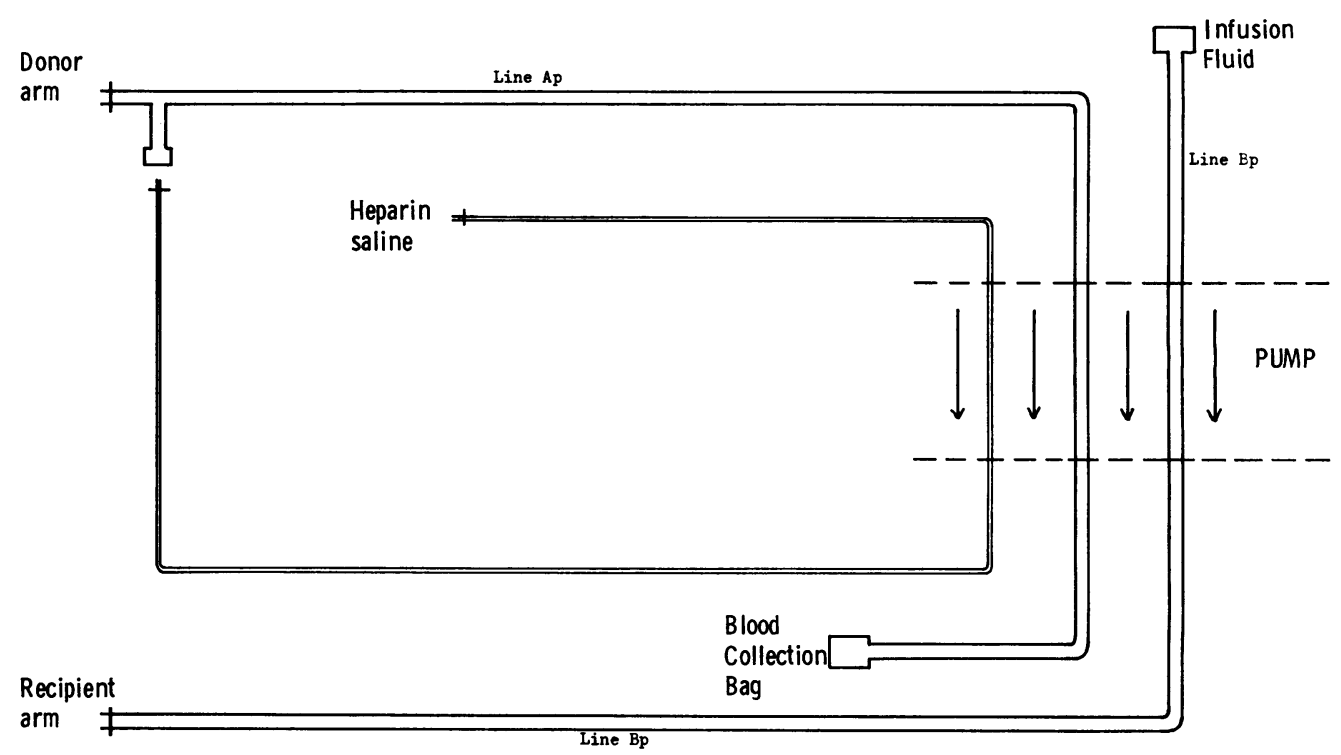

Fig. 2 Basic flow diagram for plasmapheresis exchange transfusion.

Requests for reprints to: Dr Robert Slade, Department of Haematology, The Leicester Royal Infirmary, Leicester LE1 $5 \mathrm{WW}$

\section{A new approach to the preparation of bicarbonate solution for use in virus isolation media}

A. G. HIGGINSON AND R. L. C. PILE Department of
Microbiology, Royal Air Force Institute of Pathology
and Tropical Medicine, Halton, Aylesbury, Bucks, UK

The preparation of bicarbonate solution in small sterile aliquots for addition to culture media as a buffering agent is standard practice in many laboratories. It is necessary to pre-gas this solution with carbon dioxide in order to ensure a final $\mathrm{pH}$ of $7 \cdot 4$ and adequate buffering in the final medium. It is difficult and time-consuming to obtain a sufficient concentration of carbon dioxide in the solution, particularly as autoclaving the aliquots even with tight stoppering causes some loss of carbon dioxide. The need for a new and expensive regulator for the

Received for publication 13 April 1978 carbon dioxide cylinder caused us to look for a more efficient method of gassing. An ordinary 1.8 litre Sparklets soda syphon was found to be a cheaper alternative, to save a considerable amount of technician time, and to be more effective, giving an increased shelf-life to the aliquots.

\section{Material and methods}

BICARBONATE SOLUTION

Sodium hydrogen carbonate $88 \mathrm{~g}$

$0 \cdot 2 \%$ Phenol red $^{1} \quad 100 \mathrm{ml}$

Deionised water $\quad 1900 \mathrm{ml}$

The sodium hydrogen carbonate and $0.2 \%$ phenol red are placed in a flask and made up to 2 litres with deionised water. This is used to fill the 1.8 litre Sparklets soda syphon, which is then gassed using two Sparklets carbon dioxide bulbs, and the contents are shaken vigorously for two

$10.2 \%$ phenol red solution is made by dissolving $100 \mathrm{mg}$ of phenol red in $5 \mathrm{ml} 0.05$ molar sodium hydroxide and diluting to $50 \mathrm{ml}$ with deionised water. 
minutes after charging with each bulb. The contents are then distributed in $10 \mathrm{ml}$ amounts into 180 universal bottles, which are then tightly capped and autoclaved at $10 \mathrm{psi}$ for 15 minutes. These aliquots are then stored at $4^{\circ} \mathrm{C}$ until required. A spare universal container cap fitted onto the Sparklets spout greatly assists the dispensing of the solution by pr-venting splash back. The final $\mathrm{pH}$ of these aliquots is $7 \cdot 5$.

\section{CELL LINES}

For routine viral culture we use three main cell lines. These are: (a) primary African green monkey kidney cells (Flow Laboratories); (b) human embryo lung IPTM 77 (Royal Air Force Institute of Pathology and Tropical Medicine); (c) HeLa cells (Flow Laboratories).

The bicarbonate aliquots are used to make up growth and maintenance media for these cell lines.

\section{CULTURE MEDIA}

For use with monkey kidney and HeLa cells Growth medium:

Eagles MEM (Burroughs Wellcome); $4 \%$ calf serum; $2 \%$ bicarbonate solution; $1 \%$ penicillin and streptomycin.

\section{Maintenance medium:}

Medium 199 (Burroughs Wellcome); $1 \%$ calf serum; $2 \%$ bicarbonate solution; $1 \%$ penicillin and streptomycin.

For use with human embryo lung IPTM 77

As above, except $8 \%$ calf serum is used in the growth medium and 3\% calf serum in the maintenance medium.

\section{Results}

The bicarbonate solution has so far remained stable for four months. Using this solution the final $\mathrm{pH}$ of the growth and maintenance media is $7 \cdot 4$, and culture of the three cell lines has been satisfactory. Since using this method we have successfully isolated Herpes simplex, Echo 15, Echo 19, Coxsackie B 1-6, polio and mumps viruses, and performed satisfactorily in National Quality Controls.

\section{Discussion}

The bicarbonate ion is the most important buffering ion in most tissue culture media, and most systems require some control of carbon dioxide tension in the gas phase and in the medium (Paul, 1973). Harris (1954) suggests that for the growth of some cell types the bicarbonate ion is essential. It is standard practice, therefore, to add bicarbonate solution to tissue culture media. Sodium bicarbonate converts to sodium carbonate with the release of carbon dioxide, particularly when autoclaved, and it is necessary to saturate the solution with carbon dioxide to convert all the carbonate to bicarbonate and keep it in that state. Hitherto we have saturated our bicarbonate solution with carbon dioxide by bubbling carbon dioxide through it in two stages, firstly in bulk and secondly when it has been dispensed into $10 \mathrm{ml}$ aliquots in universal bottles. This method results in considerable loss of medium through excessive frothing and is very time-consuming and tedious. The first stage takes 60 minutes and needs constant attention because of frothing, and the second stage takes 30 seconds/bottle (a further 90 minutes for 180 aliquots). Because of the limited amount of carbon dioxide that can be dissolved in this way we found that the shelf-life of these solutions, even in tightly stoppered bottles, rarely exceeds one month. Using the soda syphon modification we can gas 180 aliquots in four minutes and, because of the extended shelf-life, batches need be prepared only infrequently and none has had to be discarded, thereby eliminating wastage of material and labour. We have shown that a solution produced by our method is easier and quicker to prepare, has a superior shelf-life, and is satisfactory in use.

We thank Chief Technician J. Chater and Sergeant T. C. Simons.

\section{References}

Harris, M. (1954). The role of bicarbonate for outgrowth of chick heart fibroblasts in vitro. Journal of Experimental Zoology, 125, 85-98.

Paul, J. (1973). Cell and Tissue Culture, 4th edition, p. 57. Churchill Livingstone, Edinburgh and London.

Requests for reprints to: Squadron Leader A. G Higginson, Old Microbiology Department, RAF Institute of Pathology and Tropical Medicine, Halton, Aylesbury, Bucks HP22 5PG 